\title{
Potentiation of Antibiofilm Activity of Amphotericin B by Superoxide Dismutase Inhibition
}

\author{
Katrijn De Brucker, ${ }^{1}$ Anna Bink, ${ }^{1}$ Els Meert, ${ }^{1}$ Bruno P. A. Cammue, ${ }^{1,2}$ and Karin Thevissen ${ }^{1}$ \\ ${ }^{1}$ Centre of Microbial and Plant Genetics, KU, Leuven, 3001 Heverlee, Belgium \\ ${ }^{2}$ CMPG, Kasteelpark Arenberg 20, 3001 Heverlee, Belgium \\ Correspondence should be addressed to Bruno P. A. Cammue; bruno.cammue@biw.kuleuven.be
}

Received 8 May 2013; Accepted 23 July 2013

Academic Editor: Cristina Mazzoni

Copyright (C) 2013 Katrijn De Brucker et al. This is an open access article distributed under the Creative Commons Attribution License, which permits unrestricted use, distribution, and reproduction in any medium, provided the original work is properly cited.

This study demonstrates a role for superoxide dismutases (Sods) in governing tolerance of Candida albicans biofilms to amphotericin B (AmB). Coincubation of C. albicans biofilms with AmB and the Sod inhibitors N,N'-diethyldithiocarbamate (DDC) or ammonium tetrathiomolybdate (ATM) resulted in reduced viable biofilm cells and increased intracellular reactive oxygen species levels as compared to incubation of biofilm cells with AmB, DDC, or ATM alone. Hence, Sod inhibitors can be used to potentiate the activity of $\mathrm{AmB}$ against C. albicans biofilms.

\section{Introduction}

Candida albicans biofilms are responsible for device-related infections in most nosocomial diseases [1]. Such infections are particularly serious because biofilm-associated Candida cells are relatively resistant to a wide spectrum of antifungal drugs, including amphotericin B (AmB) [2]. The cause of this increased resistance is not yet fully elucidated but could be due to a combined action of different mechanisms including (i) expression of resistance genes, (ii) drug binding to the extracellular matrix, (iii) the change in membrane composition, or (iv) the presence of persister cells, which are cells that can survive high doses of an antimicrobial agent [3]. Due to this increased resistance, biofilm eradication and treatment of associated infections are challenging. The recalcitrance to antifungal therapy remains the biggest threat to patients with fungal biofilms and is an increasingly significant clinical problem [4]. Understanding the role of fungal biofilms during infection should help the clinical management of these recalcitrant infections. Until now, no vaccines are available to combat fungal infections, despite the considerable growth in the research field [5]. Therefore, the use of antimycotics is currently the only clinical solution for these infections. Among the current antimycotics in clinical use, only the liposomal formula of $\mathrm{AmB}$ and echinocandins has shown consistent in vitro and in vivo activity against $C$. albicans biofilms [6-8]. AmB is a fungicidal polyene and, apart from its interaction with ergosterol and subsequent pore formation, induces accumulation of reactive oxygen species (ROS) and apoptosis in planktonic and biofilm C. albicans cells $[9,10]$. Despite its high efficacy as an antimycotic, the effective concentrations of $\mathrm{AmB}$ required for elimination of Candida biofilms are often hepatoxic and/or nephrotoxic $[11,12]$. Therefore, in order to improve the potential of AmB for treatment of such biofilms, it is recommended to search for new approaches in which the effective concentration of $\mathrm{AmB}$ against $C$. albicans biofilms and consequently also its negative side effects are reduced.

In this study, we aimed at identifying compounds that lead to increased antibiofilm activity of AmB. Recently, we reported that superoxide dismutases (Sods) are involved in $C$. albicans biofilm persistence to the ROS-inducing antifungal miconazole. C. albicans contains 6 different Sods, which are involved in the detoxification of ROS by converting $\mathrm{O}_{2}{ }^{-}$ into molecular oxygen and hydrogen peroxide [13, 14]. Sod1, Sod4, Sod5, and Sod6 of C. albicans are Cu,Zn-containing superoxide dismutases [14] that can be inhibited using the Cu,Zn-Sod inhibitor N, $\mathrm{N}^{\prime}$-diethyldithiocarbamate (DDC), 
which chelates copper [15]. We previously demonstrated that this inhibitor potentiates the activity of miconazole against C. albicans persister cells within biofilms, thereby allowing ROS build-up and intensive killing of the persister cells [16]. Ammonium tetramolybdate (ATM) is another copper chelator which is used in clinical applications. For example, ATM is used therapeutically in the treatment of copper metabolism disorders (e.g., Wilson's disease) where it reduces copper adsorption or removes excess copper from the body [17-19]. ATM inhibits activities of a variety of $\mathrm{Cu}$-utilizing enzymes, including $\mathrm{Cu}, \mathrm{Zn}$-Sod1 [20-22]. In the present study, we investigated a putative effect of DDC or ATM on the activity of AmB against C. albicans biofilms and planktonic cells.

\section{Materials and Methods}

2.1. Materials, Yeast Strains, Plasmids, and Growth Media. C. albicans CA-IF100 [13], C. albicans clinical isolates F17, G6 [23], and 2CA [16] were used in this study. Growth medium was YPD ( $1 \%$ yeast extract, $2 \%$ peptone, and $2 \%$ glucose) and SC (1\% CSM, complete amino acid supplement mixture, $1 \%$ YNB, yeast nitrogen base; $2 \%$ glucose). N-N ${ }^{\prime}$-diethyldithiocarbamate $(\mathrm{DDC})($ stock $=1 \mathrm{M}$ in water), ammonium tetrathiomolybdate (ATM) (stock = $1 \mathrm{M}$ in DMSO) and $\mathrm{AmB}$ (stock $=5 \mathrm{mM}$ in DMSO), were purchased from Sigma (St. Louis, MO, USA). DHE was purchased from Life technologies (Paisley, UK). Phosphatebuffered saline (PBS) was prepared by combining $8 \mathrm{gl}^{-1}$ $\mathrm{NaCl}, 0.2 \mathrm{gl}^{-1} \mathrm{KCl}, 1.44 \mathrm{gl}^{-1} \mathrm{Na}_{2} \mathrm{HPO}_{4}$, and $0.24 \mathrm{gl}^{-1} \mathrm{KH}_{2} \mathrm{PO}_{4}$ ( $\mathrm{pH} 7.4)$.

2.2. Drug Susceptibility Testing against Planktonic C. albicans Cells. Overnight cultures of C. albicans were washed in PBS and diluted in SC medium to $1 \times 10^{6}$ cells $/ \mathrm{mL}$. Cultures were treated with $0.156 \mu \mathrm{M}$ AmB, $10 \mathrm{mM}$ DDC or $0.156 \mu \mathrm{M}$ $\mathrm{AmB}$, and $10 \mathrm{mM} \mathrm{DDC}$ and incubated for 24 hours at $37^{\circ} \mathrm{C}$. DMSO (2\%) was used as a control treatment. Next, cells were diluted in PBS and plated on YPD agar plates. Afterwards, the number of colony-forming units was determined and the percentage of surviving C. albicans cells was calculated relative to the DMSO control treatment.

2.3. Drug Susceptibility Testing on C. albicans Biofilms. The activity of $\mathrm{AmB}$ (final DMSO concentration = 2\%) in the absence or presence of $10 \mathrm{mM}$ DDC or $10 \mathrm{mM}$ ATM against $16 \mathrm{~h}$ old C. albicans biofilms was assessed in PBS as described previously [16]. DMSO (2\%) was used as a control treatment. Briefly, after incubation for $24 \mathrm{~h}$, biofilms were washed, resuspended in PBS by vigorous vortexing, and plated on YPD agar plates. The fraction of viable biofilm cells was determined by counting the colonies and calculating the percentage of surviving Candida cells, relative to the control treatment.

2.4. ROS Accumulation Assay in C. albicans Biofilm Cells. Quantification of ROS using $2^{\prime}, 7^{\prime}$-dichlorodihydrofluorescein diacetate (DCFHDA) was performed as previously described [16]. Quantification of ROS was additionally determined using dihydroethidium (DHE). To this end, C. albicans biofilms were treated with $1 \mu \mathrm{M}$ AmB in presence or absence of $10 \mathrm{mM}$ DDC or $10 \mathrm{mM}$ ATM (final DMSO concentration = $2 \%)$. As a control treatment, $2 \%$ DMSO was used. After $24 \mathrm{~h}$ incubation at $37^{\circ} \mathrm{C}$, biofilms were washed and resuspended in PBS by vigorous vortexing. A sample was taken for colony counting, after which the biofilm cells were incubated for 20 minutes at $37^{\circ} \mathrm{C}$ with $20 \mu \mathrm{M}$ DHE. After washing, fluorescence was measured $(510 \mathrm{~nm} / 595 \mathrm{~nm})$ using a fluorescence spectrometer and values were normalized to the number of CFUs.

2.5. Statistical Analysis. Statistical analysis was performed using unpaired $t$-test. Differences were considered significant if ${ }^{*} P<0.05 ;{ }^{* *} P<0.01 ;{ }^{* *} P<0.001$. Data of all experiments are represented by the mean \pm SEM.

\section{Results and Discussion}

3.1. DDC Increases the Antibiofilm Activity of $A m B$ against C. albicans. First we investigated the effect of $\mathrm{N}-\mathrm{N}^{\prime}$ diethyldithiocarbamate (DDC) on the activity of Amphotericin B (AmB) against C. albicans CA_IF-100 biofilms. To this end, a concentration of $A m B$ that had no significant effect on the viability of $C$. albicans biofilm cells was used. Treatment of $C$. albicans biofilms with $1 \mu \mathrm{M}$ AmB did not result in a statistically significant reduction of viable biofilm cells compared to control treatment (Figure 1). We used these concentrations of $\mathrm{AmB}$ to further investigate the potential of DDC on potentiating the antibiofilm activity of AmB against C. albicans biofilms. Since Lushchak and colleagues previously demonstrated that treatment of Saccharomyces cerevisiae with DDC caused a dose-dependent inhibition of Sod activity in vivo, with $75 \%$ inhibition occurring at $10 \mathrm{mM}$ DDC [15], we used a similar concentration in our experiments. Coincubation of $C$. albicans biofilms with $1 \mu \mathrm{M}$ $\mathrm{AmB}$ and $10 \mathrm{mM}$ DDC resulted in an approximately 10,000fold reduction of viable biofilm cells as compared to $\mathrm{AmB}$ or DDC treatment alone. More specifically, treatment of $C$. albicans biofilms with $1 \mu \mathrm{M} \mathrm{AmB}$ and $10 \mathrm{mM}$ DDC resulted in only $0.008 \pm 0.002 \%$ viable biofilm cells $(P<0.001)$, whereas treatment of biofilms with $1 \mu \mathrm{M} \mathrm{AmB}$ alone resulted in 84.80 $\pm 9.66 \%$ viable biofilm cells. Treatment of $C$. albicans biofilms with $10 \mathrm{mM}$ DDC alone resulted only in a 2 -fold reduction of the viable biofilm cells $(P<0.05)$ (Figure 1$)$, pointing to a clearly enhanced antibiofilm activity of AmB when combined with DDC.

3.2. The Antibiofilm Activity of $A m B$ against $C$. albicans Clinical Isolates is Enhanced by DDC. To evaluate the above findings further, we assessed the antibiofilm activity of AmB and DDC using 3 C. albicans clinical isolates. Clinical isolates F17 and G6 are characterized by increased levels of AmBtolerant persisters [23] whereas strain 2CA was isolated from the voice prosthesis of different laryngectomized patients [16]. The percentage of viable biofilm cells upon treatment of C. albicans F17, G6, or 2CA biofilms is represented in 


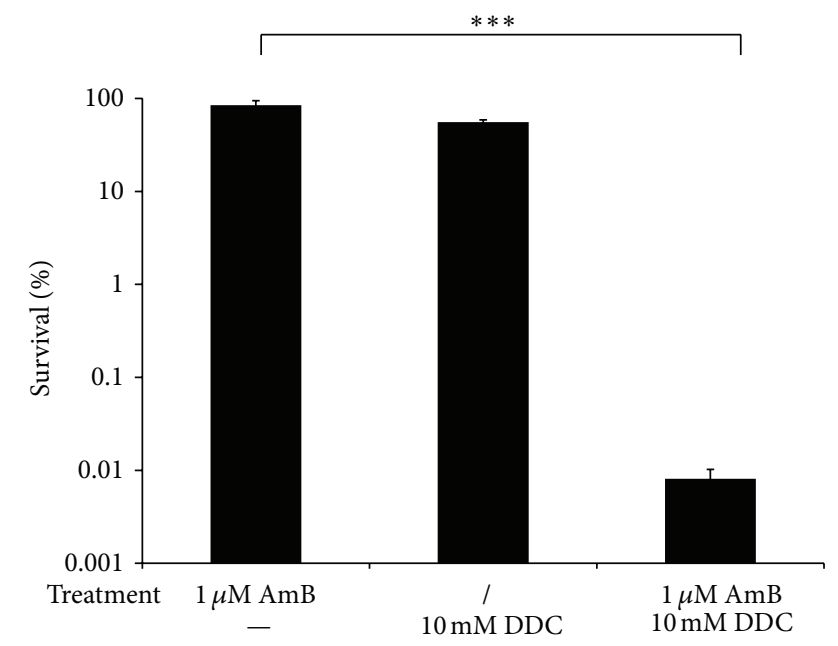

FIGURE 1: Effect of N-N'-diethyldithiocarbamate (DDC) on AmBtolerant cells in C. albicans CA-IF100 biofilms. Biofilms were treated with or without (/) $1 \mu \mathrm{M} \mathrm{AmB}$ in presence or absence (-) of $10 \mathrm{mM}$ DDC. After 24 hours, biofilms were washed with PBS and the percentage survival of $C$. albicans cells, relative to the control treatment ( $2 \% \mathrm{DMSO})$, was determined by plating the biofilm cells on YPD plates. Data represent the mean and SEM for one representative experiment out of two, each consisting of triplicate measurements. ${ }^{* * *} P<0.001$.

Figure 2. Coincubation of F17 or G6 biofilms with $1 \mu \mathrm{M}$ $\mathrm{AmB}$ and $10 \mathrm{mM}$ DDC resulted in an approximately 10,000fold reduction of viable biofilm cells as compared to AmB treatment alone. More specifically, treatment of biofilms of F17 or G6 with $1 \mu \mathrm{M} \mathrm{AmB}$ and $10 \mathrm{mM}$ DDC resulted in only $0.009 \pm 0.002 \%(P<0.001)$ or $0.01 \pm 0.004 \%(P<$ 0.01 ) of viable biofilm cells, respectively, whereas treatment of biofilms of F17 or G6 with $1 \mu \mathrm{M}$ AmB alone had no effect on the viability of these biofilm cells. Treatment of these biofilms with $10 \mathrm{mM}$ DDC alone resulted in a 3-fold reduction of viable biofilm cells $(P<0.05)$ (Figure 2$)$. Treatment of $2 \mathrm{CA}$ biofilms with $1 \mu \mathrm{M} \mathrm{AmB}$ and $10 \mathrm{mM}$ DDC resulted in an approximately 3 -fold reduction of viable cells compared to AmB treatment alone. More specifically, coincubated 2CA biofilms resulted in $20.6 \pm 2.6 \%(P<0.01)$ of viable cells, whereas treatment of these biofilms with $1 \mu \mathrm{M}$ AmB alone resulted in $66.8 \pm 5.7 \%$ of viable cells. Treatment of $2 \mathrm{CA}$ biofilms with $10 \mathrm{mM}$ DDC alone resulted in $82.7 \pm 11.5 \%$ viable cells (Figure 2). These results confirm that inhibition of Sod activity by DDC can potentiate the antibiofilm activity of $\mathrm{AmB}$ against various $C$. albicans clinical isolates. The extent of potentiation of the $\mathrm{AmB}$ antibiofilm activity seems strain dependent.

3.3. Potentation of Antifungal Activity of $A m B$ by $D D C$ is Not Biofilm Specific. The activity of $\mathrm{AmB}$ in the presence of DDC against planktonic C. albicans CA_IF-100 cells was determined. Also here, concentrations of $\mathrm{AmB}(0.156 \mu \mathrm{M})$ and DDC $(1.25 \mathrm{mM})$ were used that had no or limited effect on the viability of planktonic C. albicans cells. The percentage of viable C. albicans planktonic cells after treatment with or

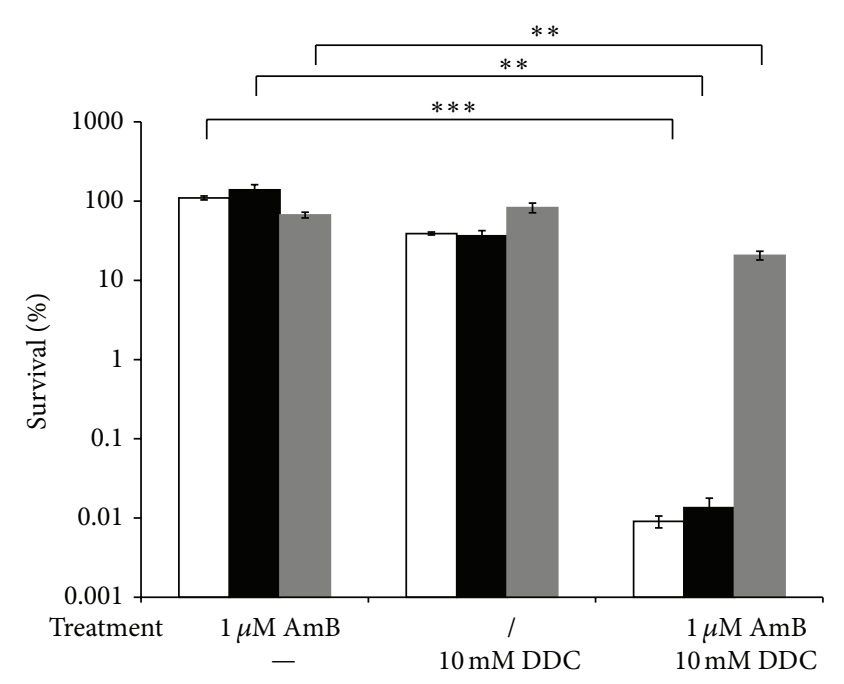

FIgURE 2: Effect of DDC on AmB-tolerant cells in biofilms of $C$. albicans clinical isolates F17, G6, and 2CA. C. albicans biofilms of F17, G6, or 2CA were treated with or without (/) $1 \mu \mathrm{M} \mathrm{AmB} \mathrm{in}$ presence or absence (-) of $10 \mathrm{mM}$ DDC. Afterwards, biofilms were washed with PBS and survival of Candida cells was determined by plating the biofilm cells on YPD plates. The AmB-tolerant fraction in presence or absence of $10 \mathrm{mM}$ DDC was determined relative to DMSO treatment. Data represent the mean and SEM for one representative experiment out of two, each consisting of triplicate measurements. F16 (white bars), G6 (black bars), 2CA (grey bars). ${ }^{* *} P<0.01 ;{ }^{* * *} P<0.001$.

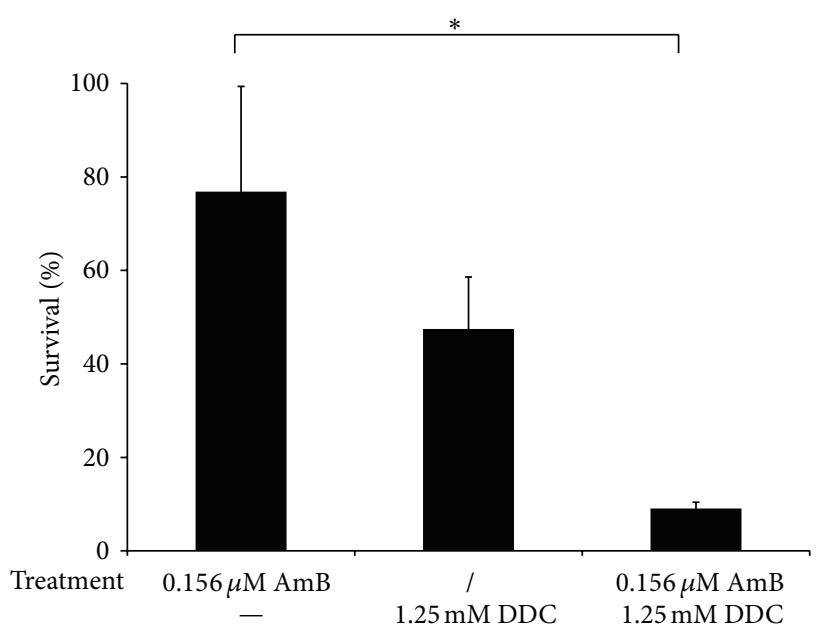

FIGURE 3: Effect of combined treatment of DDC and $\mathrm{AmB}$ on planktonic C. albicans cell cultures. Planktonic C. albicans cultures were treated with or without (/) $0.156 \mu \mathrm{M} \mathrm{AmB}$ in presence of absence (-) of $1.25 \mathrm{mM}$ DDC for 24 hours and afterwards plated on YPD plates. The percentage survival relative to the control treatment ( $2 \%$ DMSO) is shown. Data represent the mean of 2 independent biological experiments, each consisting of two measurements. ${ }^{*} P<$ 0.05 .

without $\mathrm{AmB}$ in presence or absence of DDC is shown in Figure 3. Combined treatment of $\mathrm{AmB}$ and $\mathrm{DDC}$ resulted in an 8 -fold reduction of the percentage of viable $C$. albicans cells compared to planktonic cells treated with AmB only. The percentage of viable planktonic cells after combined 


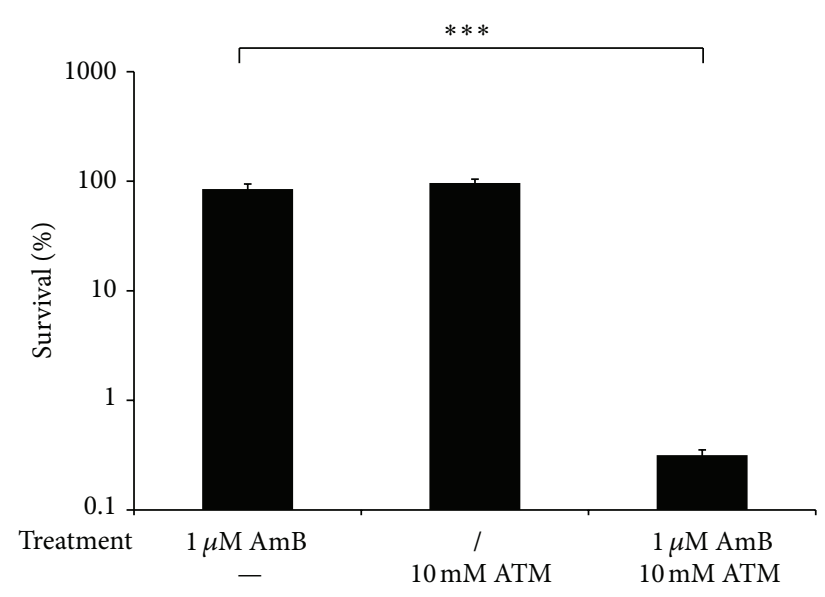

(a)

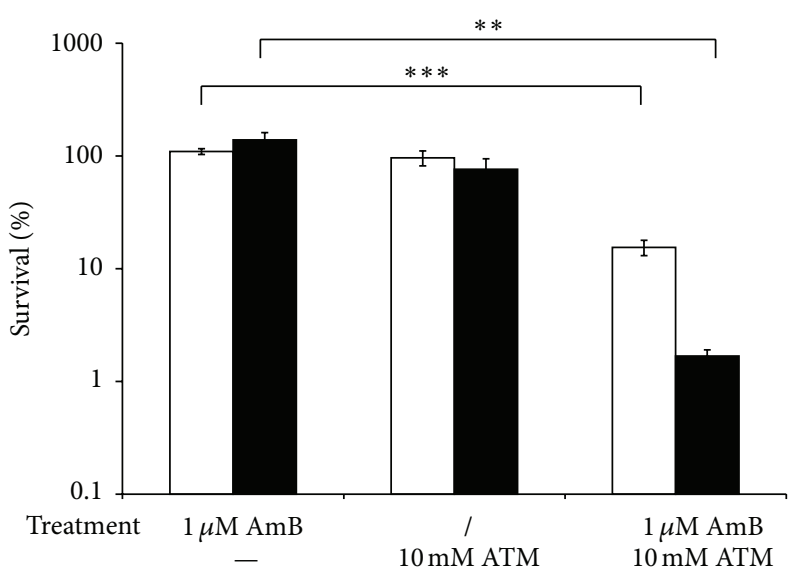

(b)

FIGURE 4: Effect of ATM on AmB-tolerant cells in biofilms of C. albicans CA-IF100 (a) and clinical isolates F17 and G6 (b). C. albicans biofilms were treated without (/) or with $1 \mu \mathrm{M} \mathrm{AmB}$ in the absence (-) or presence of $10 \mathrm{mM}$ ATM. Afterwards, biofilms were washed with PBS and survival of Candida cells was determined by plating the biofilm cells on YPD plates. The AmB-tolerant fraction in presence or absence of $10 \mathrm{mM}$ DDC was determined relative to DMSO treatment. Data represent the mean and SEM for one representative experiment out of two, each consisting of triplicate measurements. (a) CA-IF100 and (b) clinical isolates F17 (white bars) and G6 (black bars). ${ }^{* *} P<0.01$; ${ }^{* * *} P<0.001$.

treatment of $\mathrm{AmB}$ and $\mathrm{DDC}(9 \pm 1 \%)$ was significantly reduced $(P<0.05)$ compared to treatment with AmB (77 $\pm 20 \%)$ or DDC $(47 \pm 11 \%)$ alone (Figure 3$)$. This result shows that DDC-dependent potentiation of the activity of $\mathrm{AmB}$ is not biofilm specific, as DDC also potentiates the activity of $\mathrm{AmB}$ against planktonic cells, albeit to a lesser extent.

\subsection{ATM, a Clinical Used Copper Chelator, Increases the Activ-} ity of AmB against C. albicans Biofilm Cells. As DDC is associated with neurotoxicity [24], which might limit its clinical potential, we also determined the effect of ATM, a therapeutically used copper chelator $[17,18]$, on the antibiofilm activity of AmB. Coincubation of C. albicans CA_IF-100 biofilms with $1 \mu \mathrm{M} \mathrm{AmB}$ and $10 \mathrm{mM}$ ATM led to an approximately $300-$ fold reduction of viable biofilm cells, resulting in only 0.32 $\pm 0.04 \%(P<0.001)$ of viable biofilm cells, compared to AmB treatment alone. Treatment of C. albicans biofilms with $10 \mathrm{mM}$ ATM alone did not result in a significant reduction of the viable biofilm cells (Figure 4(a)). In addition, the percentage of viable $C$. albicans cells of two clinical isolates, F17 and G6, was also determined after treatment without or with $1 \mu \mathrm{M} \mathrm{AmB}$ in the presence or absence of $10 \mathrm{mM}$ ATM (Figure 4(b)). Co-incubation of F17 or G6 biofilms with $1 \mu \mathrm{M} \mathrm{AmB}$ and $10 \mathrm{mM}$ ATM resulted in a 7 - or 80 -fold significant reduction of viable biofilm cells, respectively, as compared to AmB treatment alone (Figure 4(b)). Treatment of biofilms of F17 or G6 with $1 \mu \mathrm{M} \mathrm{AmB}$ and $10 \mathrm{mM}$ ATM resulted in $15.47 \pm 2.40 \%(P<0.001)$ or $1.68 \pm 0.22 \%(P<$ $0.01)$ viable biofilm cells, respectively, whereas treatment of biofilms of F17 or G6 with $1 \mu \mathrm{M}$ AmB alone had no effect on the viability of these biofilm cells. Treatment of these biofilms with $10 \mathrm{mM}$ ATM alone resulted in no significant reduction of the viable biofilm cells (Figure 4(b)). These results indicate that ATM also increases the activity of AmB against clinical isolates, albeit to a lesser extent compared to the wild-type CA-IF100, in contrast to DDC. This might indicate that DDC is more effective in inhibiting Sods compared to ATM. However, additional studies are necessary to investigate this further.

\subsection{Treatment with DDC and ATM Increases Endogenous} Reactive Oxygen Species Levels in AmB-Treated Biofilms. To determine if treatment with the Sod inhibitors DDC or ATM enhances endogenous reactive oxygen species (ROS) levels in AmB-treated biofilms, the accumulation of ROS was quantified using $2^{\prime}, 7^{\prime}$-dichlorodihydrofluorescein diacetate (DCFHDA) or dihydroethidium (DHE). DCF, the conversion product of DCFHDA, indicates the presence of several types of ROS, including hydrogen peroxide and peroxyl radicals, whereas DHE is a specific superoxide detection reagent [25]. In a first series of experiments, we used DCFHDA as detection reagent. Coincubation of CA-IF100 biofilms with $\mathrm{AmB}$ and DDC resulted in significantly increased endogenous ROS levels in C. albicans biofilm cells as compared to AmB or DDC treatment alone. CA-IF100 biofilms, treated with a combination of $1 \mu \mathrm{M} \mathrm{AmB}$ and $10 \mathrm{mM}$ DDC and incubated with $\mathrm{H} 2 \mathrm{DCFA}$, resulted in an approximately 10,000-fold increase of endogenous ROS levels $(P<0.05)$, compared to biofilms treated with $\mathrm{AmB}$ or DDC alone (Figure 5(a)). Also coincubation of C. albicans biofilms with $1 \mu \mathrm{M} \mathrm{AmB}$ and $10 \mathrm{mM}$ ATM resulted in an approximately 50 -fold increase of endogenous ROS levels in C. albicans biofilm cells (Figure 5(a) $(P<0.001)$, indicating again that DDC seems more effective in inhibiting Sods compared to ATM. As Sods convert superoxide to hydrogen peroxide, we have set up additional experiments in which we specifically 


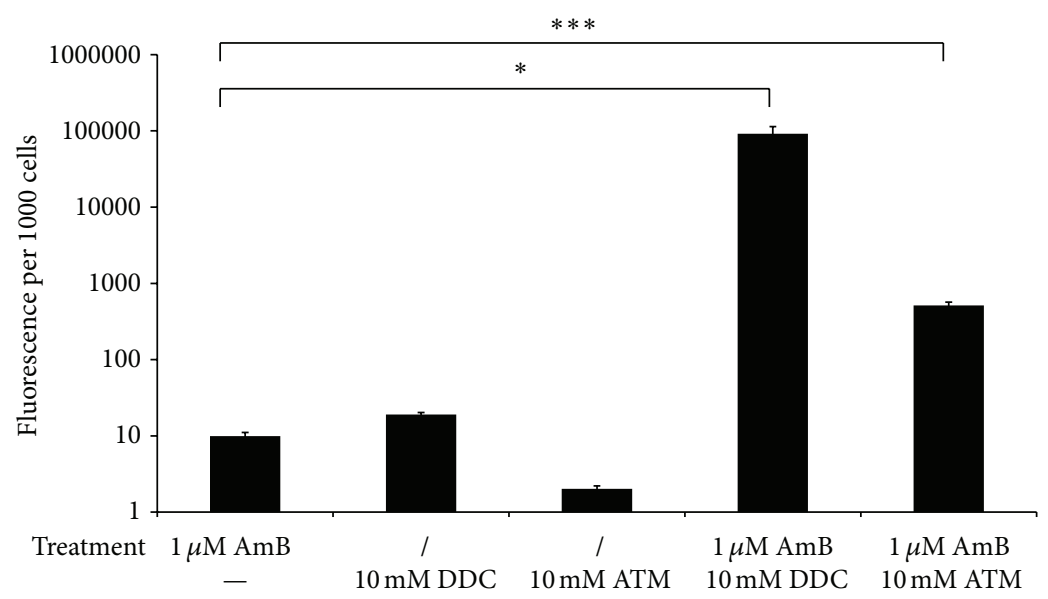

(a)

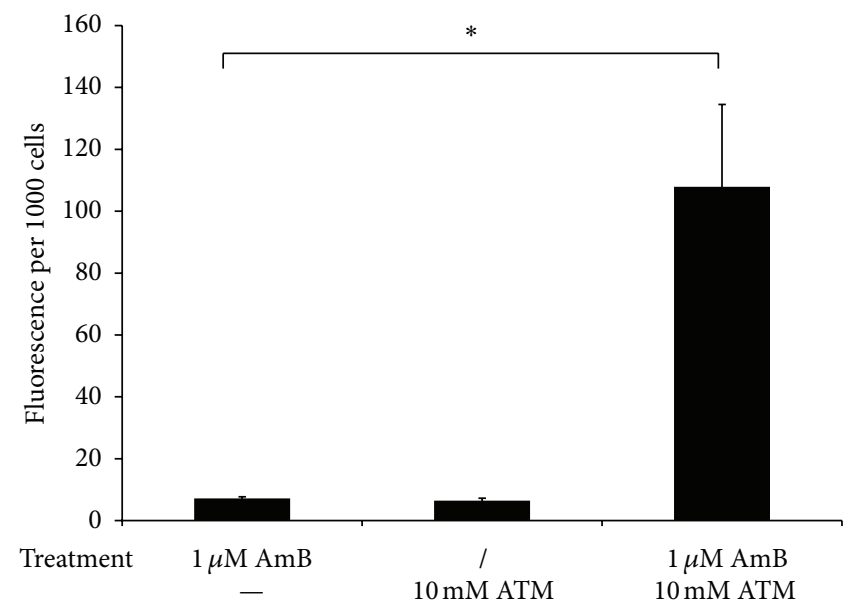

(b)

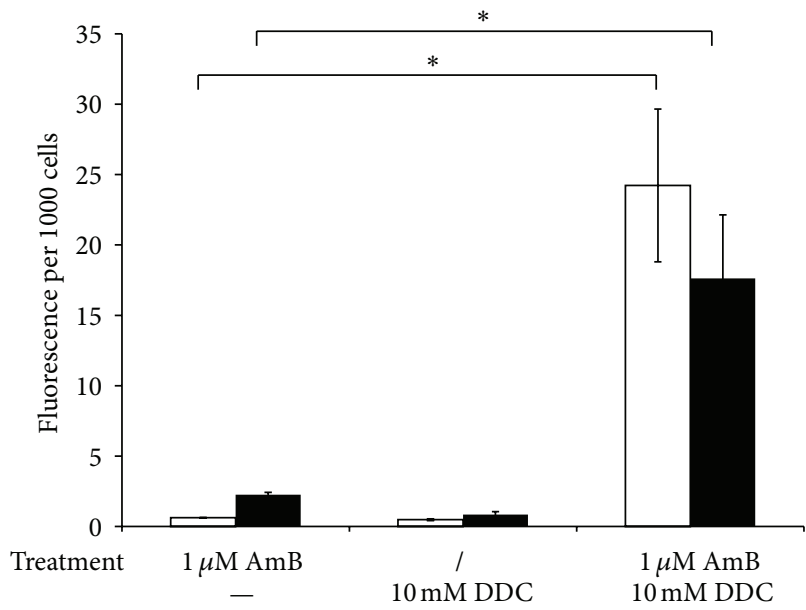

(c)

FIgURE 5: Effect of DDC and ATM on ROS levels in C. albicans biofilms. (a) C. albicans CA-IF100 biofilms were treated with or without (/) $1 \mu \mathrm{M}$ AmB in presence or absence (-) of $10 \mathrm{mM}$ DDC or $10 \mathrm{mM}$ ATM. Biofilms were washed with PBS and a sample was taken for CFU determination. Afterwards, $10 \mu \mathrm{M}$ DCFDA was added. Fluorescence was normalized to the number of CFUs after treatment. (b) and (c). Effect of DDC on peroxide levels on C. albicans biofilm cells. C. albicans biofilms were treated with or without (/) $1 \mu \mathrm{M}$ AmB in presence or absence $(-)$ of $10 \mathrm{mM}$ DDC. Biofilms were washed with PBS and a sample was taken for CFU determination. Afterwards, $20 \mu \mathrm{M}$ DHE was added. Fluorescence was normalized to the number of CFUs after treatment. (b) CA-IF100, (c) F17 (white bars), and G6 (black bars). Data represent the mean and SEM for one representative experiment out of two, each consisting of triplicate measurements. ${ }^{*} P<0.05,{ }^{* * *} P<0.001$.

monitored superoxide accumulation, using DHE staining, in C. albicans CA-IF100, G6, and F17 biofilms cells upon various treatments. Co-incubation of CA-IF100 biofilm cells with $\mathrm{AmB}$ and $\mathrm{DDC}$ resulted in an approximately 15 -fold increased superoxide accumulation $(P<0.05)$, compared to biofilms treated with $\mathrm{AmB}$ or DDC alone (Figure 5(b)). Moreover, also biofilms of clinical isolates F17 and G6, treated with $\mathrm{AmB}$ and DDC, accumulated, respectively, 40or 8-fold more superoxide compared to F17 or G6 biofilms treated with AmB alone $(P<0.05)$ (Figure 5(c)). These results show that inhibition of Sod activity by DDC in the presence of $A m B$ results in a significantly increased superoxide accumulation. Hence, it seems that AmB specifically induces superoxide as a means to kill fungal cells, including biofilm cells.

\section{Conclusions}

All above data indicate that, in C. albicans biofilm cells, Sods are not only involved in protection of $C$. albicans biofilms to miconazole [16] but also to $\mathrm{AmB}$, probably via detoxification of AmB-induced superoxide.

These results are in line with results of Seneviratne and coworkers [26]. They demonstrated that C. albicans biofilm formation is associated with increased antioxidative capacities. Several proteins involved in oxidative stress defenses, including thioredoxin peroxidase and alkyl hydroperoxide reductase, are upregulated in biofilms, which may contribute to the higher resistance to ROS-inducing antifungals like $\mathrm{AmB}$ and miconazole [25]. In addition, several reports document the possibility of enhancing the fungicidal activity 
of ROS-inducing antifungals by targeting the oxidative stress response system of fungi. For example, Kim and coworkers demonstrated that different redox-potent chemosensitizing agents like natural dihydroxybenzaldehydes, thymol, or salicylaldehyde could enhance the antifungal activity of different ROS-inducing antifungals [27-29]. One of their studies specifically demonstrates that chemically targeting the oxidative stress response system of fungi effectively augments antimycotic potency of AmB [28]. Based on our data, it seems that Sod inhibitors can reduce the antioxidative capacities of $C$. albicans biofilm cells, resulting in increased efficacy of ROS-inducing antifungals. In a report of Walker and coworkers, it was demonstrated that the combination of DDC and AmB is effective in treating systemic Candida infections [30]. We now demonstrated that the combination of $\mathrm{DDC}$ and $\mathrm{AmB}$ displayed potent in vitro activity against biofilms of various C. albicans strains, including AmBtolerant clinical isolates. However, as DDC is associated with neurotoxicity, ATM or other nontoxic and specific Sod inhibitors might lead to a novel antibiofilm combination therapy, consisting of a ROS-inducing antifungal with such inhibitor.

\section{Conflict of Interests}

The authors declare that they have no conflict of interests.

\section{Authors' Contribution}

Katrijn De Brucker and Anna Bink contributed equally to this paper.

\section{Acknowledgments}

This work was supported by grants from FWO-Vlaanderen (W0.026.11N, G.0896.10N) and the Industrial Research Fund (IOF) of KU Leuven (knowledge platform IOF/KP/11/007). Furthermore, the research leading to these results has received funding from the European Commission's Seventh Framework Programme (FP7/2007-2013) under the grant agreement COATIM (Project no. 278425). Karin Thevissen acknowledges the receipt of a postdoctoral fellowship from the Industrial Research Fund and KU Leuven (IOFm/05/022) and Anna Bink a predoctoral fellowship from FWO-Vlaanderen.

\section{References}

[1] G. Ramage, E. Mowat, B. Jones, C. Williams, and J. Lopez-Ribot, "Our current understanding of fungal biofilms fungal biofilms," Critical Reviews in Microbiology, vol. 35, no. 4, pp. 340-355, 2009.

[2] N. N. Mishra, T. Prasad, N. Sharma et al., "Pathogenicity and drug resistance in Candida albicans and other yeast species," Acta Microbiologica et Immunologica Hungarica, vol. 54, no. 3, pp. 201-235, 2007.

[3] C. J. Seneviratne, L. Jin, and L. P. Samaranayake, "Biofilm lifestyle of Candida: a mini review," Oral Diseases, vol. 14, no. 7, pp. 582-590, 2008.
[4] G. Ramage, J. P. Martínez, and J. L. López-Ribot, “Candida biofilms on implanted biomaterials: a clinically significant problem," FEMS Yeast Research, vol. 6, no. 7, pp. 979-986, 2006.

[5] A. Cassone, "Fungal vaccines: real progress from real challenges," The Lancet Infectious Diseases, vol. 8, no. 2, pp. 114-124, 2008.

[6] L. Didone, D. Oga, and D. J. Krysan, "A novel assay of biofilm antifungal activity reveals that amphotericin $\mathrm{B}$ and caspofungin lyse Candida albicans cells in biofilms," Yeast, vol. 28, no. 8, pp. 561-568, 2011.

[7] D. M. Kuhn, T. George, J. Chandra, P. K. Mukherjee, and M. A. Ghannoum, "Antifungal susceptibility of Candida biofilms: unique efficacy of amphotericin B lipid formulations and echinocandins," Antimicrobial Agents and Chemotherapy, vol. 46, no. 6, pp. 1773-1780, 2002.

[8] J. A. Shuford, M. S. Rouse, K. E. Piper, J. M. Steckelberg, and R. Patel, "Evaluation of caspofungin and amphotericin B deoxycholate against Candida albicans biofilms in an experimental intravascular catheter infection model," The Journal of Infectious Diseases, vol. 194, no. 5, pp. 710-713, 2006.

[9] R. S. Al-Dhaheri and L. J. Douglas, "Apoptosis in Candida biofilms exposed to amphotericin B," Journal of Medical Microbiology, vol. 59, no. 2, pp. 149-157, 2010.

[10] A. J. Phillips, I. Sudbery, and M. Ramsdale, "Apoptosis induced by environmental stresses and amphotericin B in Candida albicans," Proceedings of the National Academy of Sciences of the United States of America, vol. 100, no. 2, pp. 14327-14332, 2003.

[11] K. V. Clemons, J. A. Schwartz, and D. A. Stevens, “Therapeutic and toxicologic studies in a murine model of invasive pulmonary aspergillosis," Medical Mycology, vol. 49, no. 8, pp. 834847, 2011.

[12] G. P. Patel, C. W. Crank, and J. B. Leikin, "An evaluation of hepatotoxicity and nephrotoxicity of liposomal amphotericin B (L-AMB)," Journal of Medical Toxicology, vol. 7, no. 1, pp. 12-15, 2011.

[13] I. E. Frohner, C. Bourgeois, K. Yatsyk, O. Majer, and K. Kuchler, "Candida albicans cell surface superoxide dismutases degrade host-derived reactive oxygen species to escape innate immune surveillance," Molecular Microbiology, vol. 71, no. 1, pp. 240-252, 2009.

[14] M. Martchenko, A.-M. Alarco, D. Harcus, and M. Whiteway, "Superoxide dismutases in Candida albicans: transcriptional regulation and functional characterization of the hyphalinduced sod5 gene," Molecular Biology of the Cell, vol. 15, no. 2, pp. 456-467, 2004.

[15] V. Lushchak, H. Semchyshyn, O. Lushchak, and S. Mandryk, "Diethyldithiocarbamate inhibits in vivo $\mathrm{Cu}, \mathrm{Zn}$-superoxide dismutase and perturbs free radical processes in the yeast Saccharomyces cerevisiae cells," Biochemical and Biophysical Research Communications, vol. 338, no. 4, pp. 1739-1744, 2005.

[16] A. Bink, D. Vandenbosch, T. Coenye, H. Nelis, B. P. A. Cammue, and K. Thevissen, "Superoxide dismutases are involved in Candida albicans biofilm persistence against miconazole," Antimicrobial Agents and Chemotherapy, vol. 55, no. 9, pp. 40334037, 2011.

[17] G. J. Brewer, F. Askari, R. B. Dick et al., "Treatment of Wilson's disease with tetrathiomolybdate: V. control of free copper by tetrathiomolybdate and a comparison with trientine," Translational Research, vol. 154, no. 2, pp. 70-77, 2009.

[18] C. F. Mills, T. T. El-Gallad, and I. Bremner, "Effects of molybdate, sulfide, and tetrathiomolybdate on copper metabolism in 
rats," Journal of Inorganic Biochemistry, vol. 14, no. 3, pp. 189207, 1981.

[19] P. V. E. Van Den Berghe and L. W. J. Klomp, "New developments in the regulation of intestinal copper absorptionn," Nutrition Reviews, vol. 67, no. 11, pp. 658-672, 2009.

[20] M. V. Chidambaram, G. Barnes, and E. Frieden, "Inhibition of ceruloplasmin and other copper oxidases by thiomolybdate," Journal of Inorganic Biochemistry, vol. 22, no. 4, pp. 231-239, 1984.

[21] J. C. Juarez, O. Betancourt Jr., S. R. Pirie-Shepherd et al., "Copper binding by tetrathiomolybdate attenuates angiogenesis and tumor cell proliferation through the inhibition of superoxide dismutase 1," Clinical Cancer Research, vol. 12, no. 16, pp. 49744982, 2006.

[22] V. Medici and G. C. Sturniolo, "Tetrathiomolybdate, a copper chelator for the treatment of Wilson disease, pulmonary fibrosis and other indications," IDrugs, vol. 11, no. 8, pp. 592-606, 2008.

[23] M. D. LaFleur, Q. Qi, and K. Lewis, "Patients with longterm oral carriage harbor high-persister mutants of Candida albicans," Antimicrobial Agents and Chemotherapy, vol. 54, no. 1, pp. 39-44, 2010.

[24] O. M. Viquez, H. L. Valentine, K. Amarnath, D. Milatovic, and W. M. Valentine, "Copper accumulation and lipid oxidation precede inflammation and myelin lesions in $\mathrm{N}, \mathrm{N}-$ diethyldithiocarbamate peripheral myelinopathy," Toxicology and Applied Pharmacology, vol. 229, no. 1, pp. 77-85, 2008.

[25] Invitrogen, The molecular probes handbook, 11th edition, chapter 18, section 18. 2.

[26] C. J. Seneviratne, Y. Wang, L. Jin, Y. Abiko, and L. P. Samaranayake, "Candida albicans biofilm formation is associated with increased anti-oxidative capacities," Proteomics, vol. 8, no. 14, pp. 2936-2947, 2008.

[27] J. H. Kim, K. L. Chan, N. Mahoney, and B. C. Campbell, "Antifungal activity of redox-active benzaldehydes that target cellular antioxidation," Annals of Clinical Microbiology and Antimicrobials, vol. 10, article 23, 2011.

[28] J. H. Kim, N. C. Faria, M. L. Martins, K. L. Chan, and B. C. Campbell, "Enhancement of antimycotic activity of amphotericin B by targeting the oxidative stress response of Candida and Cryptococcus with natural dihydroxybenzaldehydes," Frontiers in Microbiology, vol. 3, p. 261, 2012.

[29] J. H. Kim, K. L. Chan, N. C. Faria, M. L. Martins, and B. C. Campbell, "Targeting the oxidative stress response system of fungi with redox-potent chemosensitizing agents," Frontiers in Microbiology, vol. 3, p. 88, 2012.

[30] E. M. Walker Jr., D. J. Cannon, and M. E. Reifsteck, "Effects of diethyldithiocarbamate and structural analogs in mice with systemic candidial infections," Research Communications in Chemical Pathology and Pharmacology, vol. 56, no. 2, pp. 253263, 1987. 


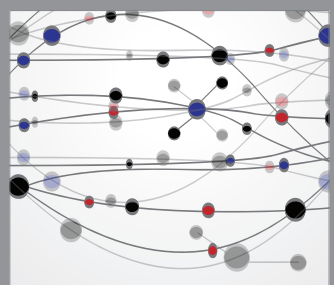

The Scientific World Journal
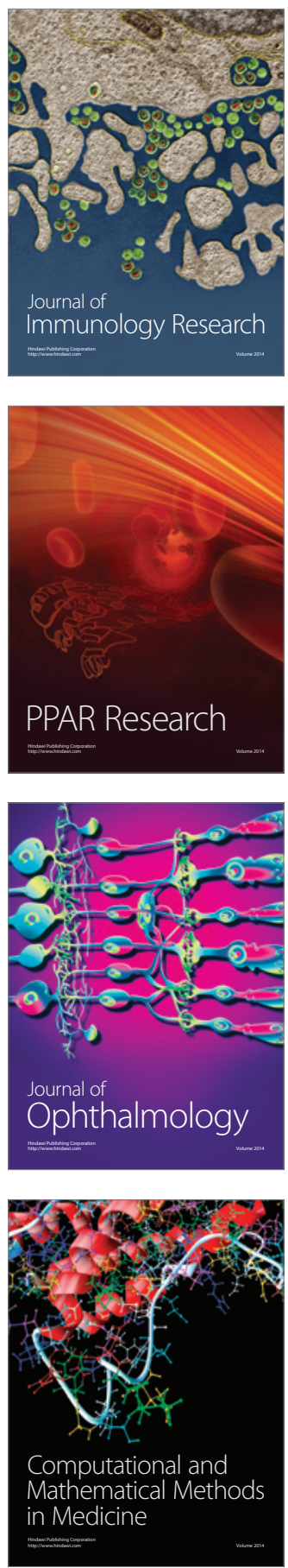

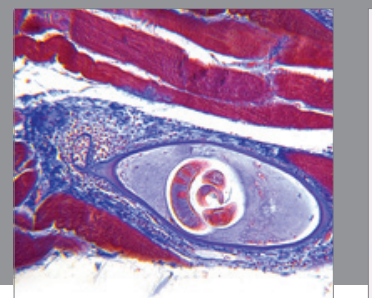

Gastroenterology

Research and Practice
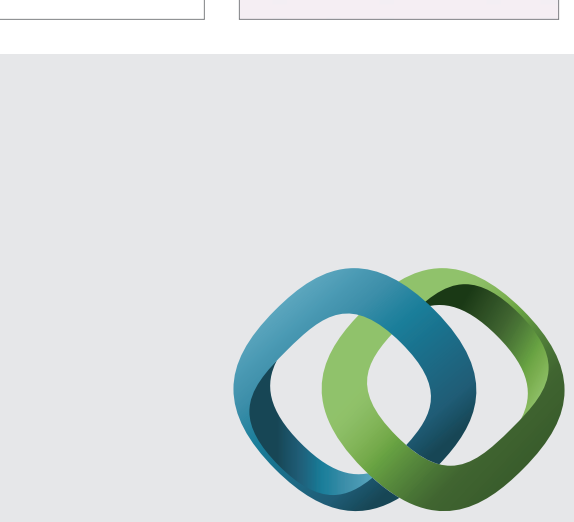

\section{Hindawi}

Submit your manuscripts at

http://www.hindawi.com
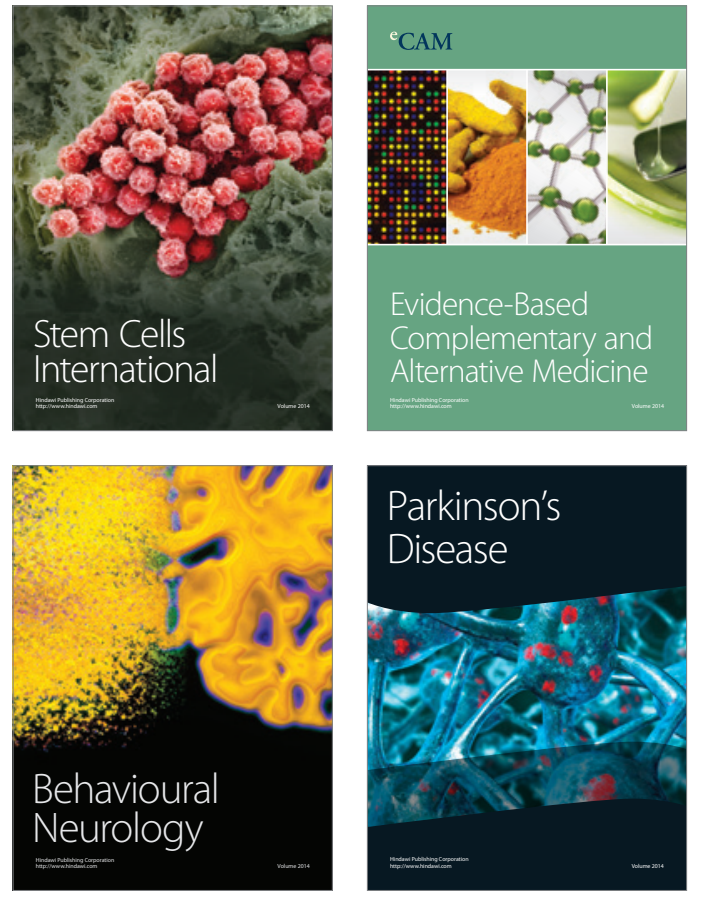
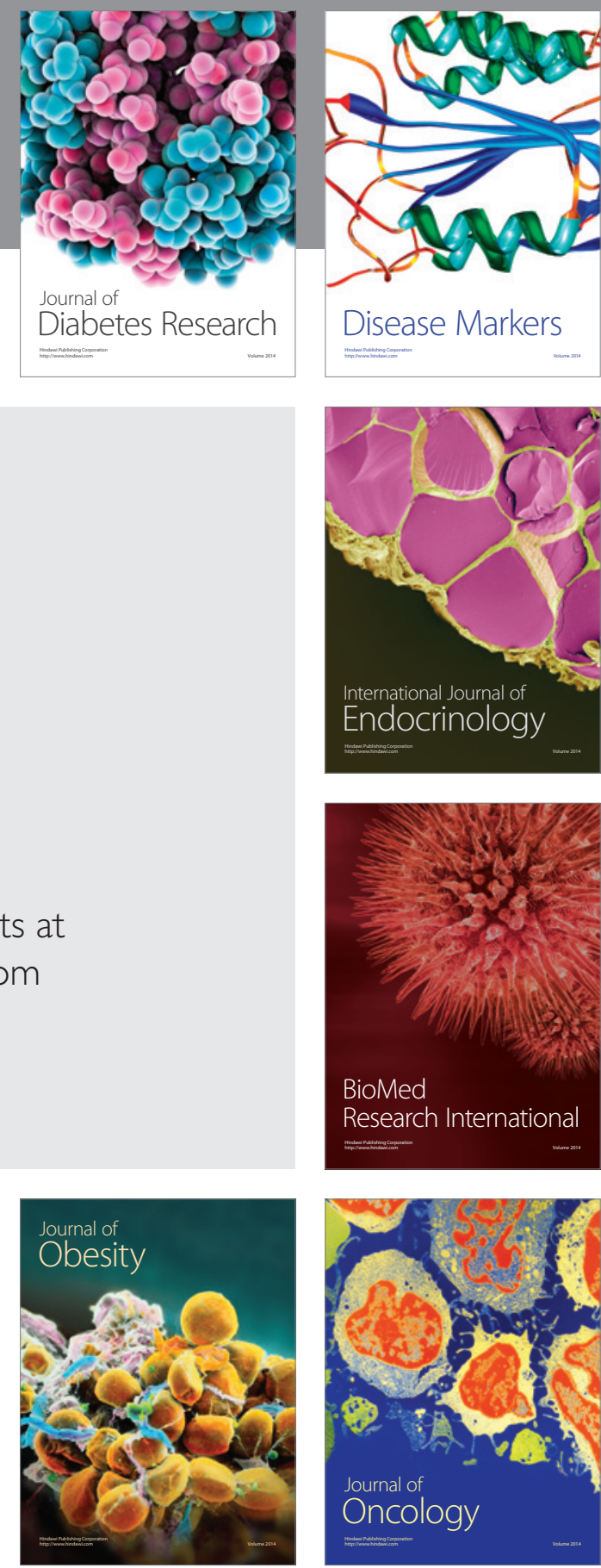

Disease Markers
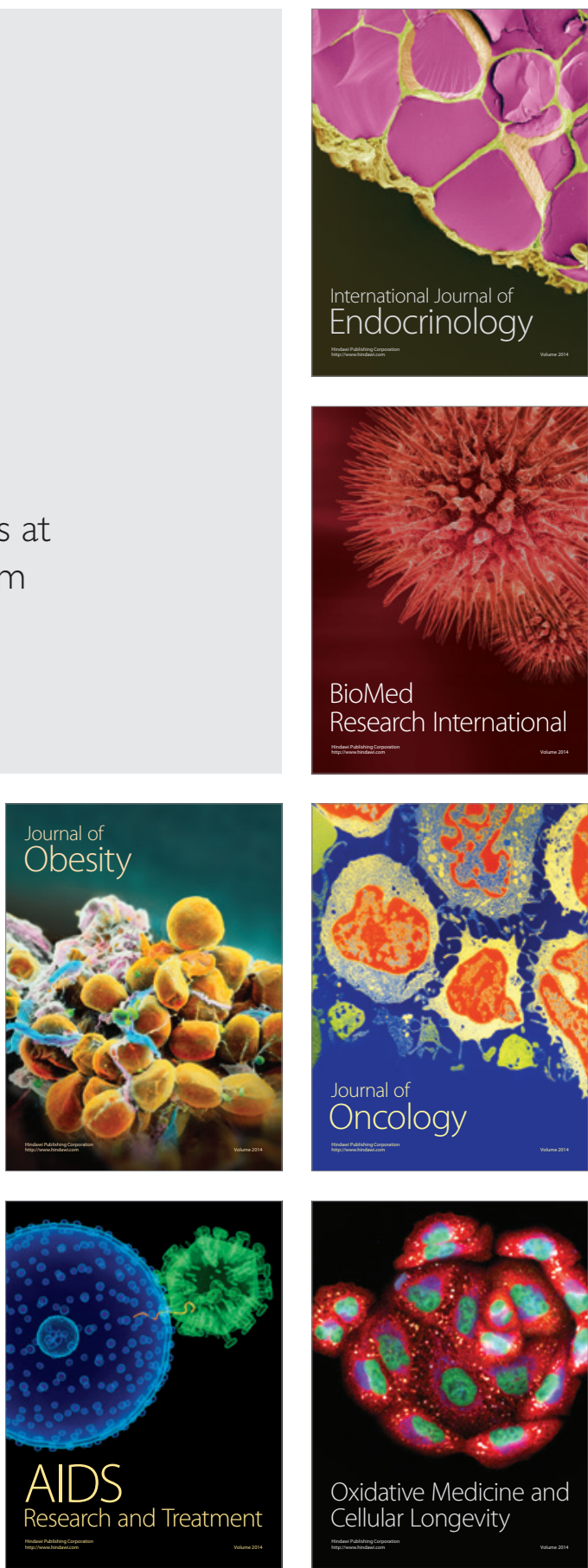